\title{
SKA Science and Coordination with Multi-messenger facilities
}

\section{A. Bonaldi et al.*, E. Keane, R. Bolton, A. Chrysostomou}

SKA Organization, Jodrell Bank, Lower Withington, Macclesfield, SK11 9DL, United Kingdom

E-mail: a.bonaldieskatelescope.org

The Square Kilometre Array (SKA) will be the world's largest radio telescope. Even in its first stage of deployment (SKA1) it will enable transformational science on a very broad range of scientific objectives. The year 2019 is pivotal for the SKA, as it sees both the transition between the design and construction phases, and between the current Organization and the SKA Observatory as an Inter-Governmental Organization.

The SKA will share the stage with several other instruments targeting other wavelengths and other messengers: LIGO, JWST, ALMA, E-ELT, CTA, ATHENA, just to cite some of them. Coordinated observations between these facilities can result in greatly enhanced scientific discoveries, and give the potential to progress our understanding of a wide range of astronomical sources and phenomena.

The New Era of Multi-Messenger Astrophysics - Asterics2019

25 - 29 March, 2019

Groningen, The Netherlands

\footnotetext{
* Speaker.
} 


\section{The SKA concept}

The SKA concept has been developed in order to answer to a set of fundamental questions:

- The Cradle of life and Astrobiology: How do planets form? Are we alone?

- Strong-field Tests of Gravity with Pulsars and Black Holes: Was Einstein right with General Relativity?

- The Origin and Evolution of Cosmic Magnetism: What is the role of magnetism in galaxy evolution and the structure of the cosmic web?

- Galaxy Evolution probed by Neutral Hydrogen: How do normal galaxies form and grow?

- The Transient radio Sky: What are Fast Radio Bursts? What haven't we discovered?

- Galaxy Evolution probed in the radio Continuum: What is the star-formation history of normal galaxies?

- Cosmology and Dark Energy: What are dark matter and dark energy? What is the large-scale structure of the Universe?

- Cosmic Dawn and the Epoch of Reionization: How and when did the first stars and galaxies form?

Such a broad range of science can be explored only by a very broad frequency range, which is probed by two separate instruments: the Low-frequency Array (SKA Low), from 50 to 350 $\mathrm{MHz}$, as a single band, and the Mid-frequency array (SKA Mid), from $350 \mathrm{MHz}$ to $24 \mathrm{GHz}$, as 5 bands, as detailed in Table 1. The former will consist of stations of log-periodic antennas and will be built in Australia; the latter will be an array of dishes built in South Africa. Both those sites have been selected as having very low Radio Frequency Interference (RFI), a feature that will be preserved in the decades to come, thanks to suitable agreements with the local governments. They already host radio astronomical facilities, including the SKA precursors MWA (Murchison Widefield Array, http://www.mwatelescope.org), ASKAP (Australian SKA Precursor, https://www.atnf.csiro.au/projects/askap/index.html) and HERA (Hydrogen Epoch of Reionization Array, http://reionization.org/) in Australia, and MeerKat (http://www.ska.ac.za/gallery/meerkat/) in South Africa.

\section{SKA project schedule}

The deployment of the telescope has been staged in two phases. In the first phase (SKA phase 1, or SKA1) 131,072 of the total 1 million elements of the Low Frequency Aperture Array, and 197 (64 of which will be integrated from Meerkat) of the total 2500 dishes of the Mid Frequency Array will be deployed. In phase 2, the remaining elements of the Low and Mid arrays will be deployed, as well as new technology (Phased Array Feeds and Mid-Frequency Aperture Array concepts are currently being developed). The deployment of receivers for the 5 bands of SKA Mid has been prioritised according to the science objectives they enable, with Bands 1,2 and 5a/5b being the top priorities (see notes in Table 1) 
Table 1: SKA frequency bands

\begin{tabular}{|l|l|l|l|c|}
\hline Telescope name & Band name & Frequency range & Bandwidth & Notes \\
\hline SKA Low & SKA Low & $50-350 \mathrm{MHz}$ & $300 \mathrm{MHz}$ & $(1)$ \\
\hline SKA Mid & Band 1 & $0.35-1.05 \mathrm{GHz}$ & $1 \mathrm{GHz}$ & $(1)$ \\
\hline & Band 2 & $0.95-1.76 \mathrm{GHz}$ & $1 \mathrm{GHz}$ & $(1)$ \\
\hline & Band 3 & $1.65-3.05 \mathrm{GHz}$ & $1 \mathrm{GHz}$ & $(2)$ \\
\hline & Band 4 & $2.80-5.18 \mathrm{GHz}$ & $2.5 \mathrm{GHz}$ & $(2)$ \\
\hline & Band 5a & $4.60-8.50 \mathrm{GHz}$ & $2 \times 2.5 \mathrm{GHz}$ & $(1)$ \\
\hline & Band 5b & $8.30-15.3 \mathrm{GHz}$ & $2 \times 2.5 \mathrm{GHz}$ & $(1)$ \\
\hline & Band 5c & $15-24 \mathrm{GHz}$ & $2 \times 2.5 \mathrm{GHz}$ & $(2)$ \\
\hline & Band A & $1.6-5.2 \mathrm{GHz}$ & $2.5 \mathrm{GHz}$ & $(2)$ \\
\hline & Band B & $4.6-24 \mathrm{GHz}$ & $2 \times 2.5 \mathrm{GHz}$ & $(2)$ \\
\hline
\end{tabular}

Notes:

${ }^{1}$ Part of the baseline design and deployed as a top priority

${ }^{2}$ Deployed as an upgrade path (to be confirmed)

\section{SKA operational model}

The SKA will have a uniquely distributed setup, with one observatory operating two telescopes on three continents for a global scientific community. The observatory's operations will be guided by principles to maximise impact and availability while minimising radio frequency interference and to ensure the data are accessible to the largest numbers of users. Of the operational principles, the most relevant for a MW/MM coordinated observations are:

- Flexible scheduling to ensure dynamic response to observing conditions and resource availability, and to provide for Targets of Opportunity and triggered events. This feature ensures that triggered observations are responded to promptly with minimum delay.

- Ability to form subarrays configured and operated independently of each other. This feature means that the SKA can be operated at any time as a set of independent, smaller telescopes. Again this feature adds a lot of flexibility to carry out several observing programmes in parallel, when the full sensitivity/imaging power of the telescope is not required. This also allows the observatory to carry out maintenance or fault response procedures (on engineering subarrays) while keeping the telescopes scientifically productive.

- Commensal observing in different forms. For instance, it will be possible for different groups to share use rights on data for different scientific objectives (data commensality). It will also be possible for pulsar timing or search projects to run concurrently with an imaging project in the same subarray (observing commensality). Finally, the use of subarrays will allow more than one project to run simultaneously on the telescope (multiplexity).

- Observatory interface to users, including data access and user support, to be provided through a network of SKA Regional Centres (SRCs).

- The observatory data products and the advanced data products that will be generated at the SDP and SRCs may also be suitable for a community much larger than the radio-astronomy one, as the interferometric data processing has already happened. 
Of relevance here are also the SKA access principles:

- Common time allocation process based on scientific merit and technical feasibility;

- Access proportional to national share in the project;

- Up to 5\% Open Time available;

- Key Science Projects to take up 50-75\% of observing time, with conventional PI-led projects taking up the remainder;

- All data to be made openly available following a proprietary period, together with tools to effectively explore the archives for the sources of interest.

These policies, and others, are as anticipated and will need to be agreed by the Observatory Council once it has formed.

\section{Some examples of MW/MM synergies}

The MW-MM information would greatly enhance the value of the surveys planned for the SKA to map the Large Scale Structures (LSS) of the Universe and probe Cosmology:

1. By measuring redshifts, therefore enabling a fully 3D continuum survey. Surveys in radio continuum are strictly 2-dimensional (2D), because the featureless frequency spectrum of radio sources does not contain any redshift information. Measuring redshifts enables us to construct a 3D survey of the LSS, which is a much more powerful cosmological probe. Optical/IR data for the same survey area (e.g. Euclid, LSST/WFIRST) would provide spectroscopic redshift counterparts for the radio catalogue. Additionally, follow-ups with an instrument like E-ELT would provide high-accuracy spectroscopic redshifts for a subset of the sources, therefore enabling an optimal calibration and correction of systematics for the sample of photometric redshifts.

2. By allowing cross-correlations of different tracers. [1] illustrates the power of cross-correlation to remove systematics for a Weak Lensing (WL) survey performed in the radio with SKA1 and in optical/IR. Moreover, cross-correlations can be sensitive to a specific component of the total signal, thus improving the constraint.

3. By identifying different radio galaxy populations. Active Galactic nuclei (AGNs), starforming galaxies, and their sub-populations are typically associated with a Dark Matter halo of different mass. Therefore, being able to separate radio galaxies into different populations effectively adds an extra layer of information and improves the cosmological constraints considerably (e.g., 2)

The mentioned surveys typically require long integration times, and their specifications will need to be agreed well in advance. The MW-MM synergy would require that the same area of the sky is mapped by different instruments, but no time synchronization would be necessary. 
Another example is the coordinated observations of Fast Radio Bursts (FRBs, 3), one of the "High Priority Science Objectives" for SKA Phase 1 [4]. It will be possible to perform 24/7 searches for FRBs with both SKA1-Low and -Mid; the search system is one low-power aspect of the rather powerful pulsar search engine (the PSS, 5). Searches for FRBs then come somewhat 'for free' and it is likely that they would 'piggy-back' most, if not all, observations at both sites. If at least some FRBs are not rendered undetectable by interstellar propagation effects at the lowest frequencies, then the gain of SKA1-Low should make it a formidable FRB search system. That SKA1-Mid will detect large numbers of FRBs is clear from the bands where the currently known population have been discovered [6]. An increasing number of FRBs have been observed to ever lower frequencies, as low as $400 \mathrm{MHz}$ [7], approaching the upper end of the SKA1-Low band. Studies of FRBs fall into roughly into two categories - looking at nearby sources to study the progenitors in detail, and studying the most distant examples to use FRBs as tools for cosmology. As recently exemplified by ASKAP, the former can be targeted by 'fly's eye' observations [8], and the latter by an incoherent or coherent beam-formed array. Both avenues require FRBs be localised in the radio, which is most effectively performed in one-shot localisation using the same data wherein the FRB is detected. Localisation can also be obtained in the radio for repeating FRBs, although at the expense of more radio observing time [9]; at present it is unclear if all FRBs repeat [10]. One this localisation is performed in the radio, and a host galaxy identified, one must perform optical/infrared observations to determine a redshift. With thousands of high-redshift FRBs expected to need such treatment the coordination of this effort needs to be carefully planned. Furthermore, in addition to this no-rush multi-wavelength follow-up one can, in the case of single-shot localisation, search for temporally-related multi-wavelength and multi-messenger signals either through 'shadowed' observations, or very rapid-response triggered observations.

\section{References}

[1] Camera, S., Harrison, I., Bonaldi, A., et al. 2017, MNRAS, 464, 4747

[2] Ferramacho, L. D., Santos, M. G., Jarvis, M. J., et al. 2014, MNRAS, 442, 2511

[3] Keane, E. F. 2018, Nature Astronomy, 2, 865

[4] Braun et al. 2015, SKA-TEL-SKO-0000007 SKA1 LEVEL 0 SCIENCE REQUIREMENTS

[5] Keane, E. F. 2018, Pulsar Astrophysics the Next Fifty Years, 158

[6] Petroff, E., Barr, E. D., Jameson, A., et al. 2016, Publications of the Astronomical Society of Australia, 33, e045

[7] Boyle, P. C., \& Chime/Frb Collaboration 2018, The Astronomer's Telegram, 11901, 1

[8] Shannon, R. M., Macquart, J.-P., Bannister, K. W., et al. 2018, Nature, 562, 386

[9] Chatterjee, S., Law, C. J., Wharton, R. S., et al. 2017, Nature, 541, 58

[10] Caleb, M., Spitler, L. G., \& Stappers, B. W. 2018, Nature Astronomy, 2, 839 